BULLETIN OF THE

AMERICAN MATHEMATICAL SOCIETY

Volume 80, Number 3, May 1974

\title{
ON DECOMPOSITIONS OF A MULTI-GRAPH INTO SPANNING SUBGRAPHS
}

\author{
BY RAM PRAKASH GUPTA ${ }^{1}$
}

Communicated by Gian-Carlo Rota, April 23, 1973

1. Let $G$ be a multi-graph, i.e., a finite graph with no loops. $V(G)$ and $E(G)$ denote the vertex-set and edge-set of $G$, respectively. For $x \in V(G)$, $d(x, G)$ denotes the degree (or valency) of $x$ in $G$ and $m(x, G)$ denotes the multiplicity of edges at $x$ in $G$, i.e., the minimum number $m$ such that $x$ is joined to any other vertex in $G$ by at most $m$ edges.

A graph $H$ is called a spanning subgraph of $G$ if $V(H)=V(G)$ and $E(H) \subseteq E(G)$. Let $k$ be any positive integer. Let

$$
\sigma: G=H_{1} \cup H_{2} \cup \cdots \cup H_{k}
$$

be a decomposition of $G$ into $k$ spanning subgraphs so that (1) $H_{1}, H_{2}, \cdots$, $H_{k}$ are spanning subgraphs of $G$; (2) $H_{1}, H_{2}, \cdots, H_{k}$ are pairwise edgedisjoint; and, (3) $\bigcup_{1 \leqq \alpha \leqq k} E\left(H_{\alpha}\right)=E(G)$. For each $x \in V(G)$, let $v(x, \sigma)$ denote the number of subgraphs $H_{\alpha}$ in $\sigma$ such that $d\left(x, H_{\alpha}\right) \geqq 1$. Evidently,

$$
v(x, \sigma) \leqq \min \{k, d(x, G)\} \quad \text { for all } x \in V(G) .
$$

2. Given a multi-graph $G$ and any positive integer $k$, we consider the problem of determining a decomposition $\sigma$ of $G$ into $k$ spanning subgraphs such that $v(x, \sigma)$ is as large as possible for each vertex $x \in V(G)$. In particular, we have proved the following two theorems.

THEOREM 2.1. If $G$ is a bipartite graph, then, for every positive integer $k$, there exists a decomposition $\sigma$ of $G$ into $k$ spanning subgraphs such that

$$
v(x, \sigma)=\min \{k, d(x, G)\} \text { for all } x \in V(G) .
$$

THEOREM 2.2. If $G$ is a multi-graph, then, for every positive integer $k$, there exists a decomposition $\sigma$ of $G$ into $k$ spanning subgraphs such that

$$
\begin{aligned}
& v(x, \sigma) \geqq \min \{k-m(x, G), d(x, G)\} \quad \text { if } d(x, G) \leqq k \\
& \geqq \min \{k, d(x, G)-m(x, G)\} \quad \text { if } d(x, G) \geqq k,
\end{aligned}
$$

for all $x \in V(G)$.

AMS (MOS) subject classifications (1970). Primary $05 \mathrm{C} 15$.

Key words and phrases. Multi-graph, bipartite graph, balanced hypergraph, spanning subgraph, cover, matching, cover index, chromatic index.

1 This research was supported in part by ONR contract N00014-67-A-0232-0016. 
Moreover, if $W \subseteq V(G)$ is such that

$$
W \cap\{x \in V(G): k-m(x, G)<d(x, G)<k+m(x, G)\}
$$

is independent, then $\sigma$ can be so chosen that, in addition to (2.2), we have

$$
\nu(x, \sigma)=\min \{k, d(x, G)\} \quad \text { for all } x \in W .
$$

3. The above theorems generalize some well-known theorems in graph theory.

Let $G$ be a multi-graph; let $H$ be a spanning subgraph of $G$. $H$ is said to be a matching of $G$ if for every vertex $x, d(x, H) \leqq 1 ; H$ is said to be a cover of $G$ if for every vertex $x, d(x, H) \geqq 1$. The chromatic index of $G$, denoted by $\chi_{1}(G)$, is defined to be the minimum number $k$ such that there exists a decomposition of $G$ into $k$ spanning subgraphs each of which is a matching of $G$. The cover index of $G$, denoted by $\kappa_{1}(G)$ is the maximum number $k$ such that there exists a decomposition of $G$ into $k$ spanning subgraphs each of which is a cover of $G$.

Theorems 3.1 and 3.2 below are obtained from Theorem 2.1 by taking $k=\max _{x \in V(G)} d(x, G)$ and $k=\min _{x \in V(G)} d(x, G)$, respectively.

THEOREM 3.1 [1]. If $G$ is a bipartite graph, then,

$$
\chi_{1}(G)=\max _{x \in V(G)} d(x, G) .
$$

THEOREM 3.2 [2]. If $G$ is a bipartite graph, then,

$$
\kappa_{1}(G)=\min _{x \in V^{(}(G)} d(x, G) .
$$

Similarly, Theorems 3.3 and 3.4 are seen to be special cases of Theorem 2.2.

THEOREM 3.3 [3], [4]. If $G$ is a multi-graph, then,

$$
\chi_{1}(G) \leqq \max _{x \in V(G)}\{d(x, G)+m(x, G)\} .
$$

THEOREM 3.4 [5]. If $G$ is a multi-graph, then,

$$
\kappa_{1}(G) \geqq \min _{x \in V(G)}\{d(x, G)-m(x, G)\} .
$$

REMARK. We have also generalized Theorem 2.1 to a theorem for balanced hypergraphs which contains as special cases some theorems due to $C$. Berge [6]. 


\section{REFERENCES}

1. D. Konig, Theorie der Graphes, Leipzig, 1935.

2. R. P. Gupta, Studies in the theory of graphs, Ph.D. Thesis, T.I.F.R., Bombay, 1967, Chapter 1; A decomposition theorem for bipartite graphs, Théorie des graphes (P. Rosentiehl, Ed.) Dunod, Paris, 1967.

3. V. G. Vizing, On an estimate of the chromatic class of a p-graph, Diskret. Analiz No. 3 (1964), 25-30. (Russian) MR 31 \#4740.

4. R. P. Gupta, Studies in theory of graphs, Chap. 2. The chromatic index and the degree of a graph, Notices Amer. Math. Soc. 13 (1966), 714, 719. Abstract \#637-14; \#66T-429.

5. - Studies in the theory of graphs, Chap. 1. The cover-index of a multi-graph (to appear).

6. C. Berge, Balanced matrices, Mathematical Programming 2 (1972), 19-31 (Proc. La Haye, 1970).

Department of Mathematics, Ohio State University, Columbus, Ohio 43210

Current address: Indian Statistical Institute, 503, Yojna Bhavan, New Delhi-1, India 